\title{
Noise-Induced Subdiffusion in Strongly Localized Quantum Systems
}

\author{
Sarang Gopalakrishnan, ${ }^{1,2}$ K. Ranjibul Islam, ${ }^{3,4}$ and Michael Knap ${ }^{5}$ \\ ${ }^{1}$ Department of Engineering Science and Physics, CUNY College of Staten Island, Staten Island, New York 10314, USA \\ ${ }^{2}$ Department of Physics and Walter Burke Institute, California Institute of Technology, Pasadena, California 91125, USA \\ ${ }^{3}$ Department of Physics and Astronomy, Texas A\&M University, College Station, Texas 77843, USA \\ ${ }^{4}$ Indian Institute of Science Education and Research-Kolkata, Mohanpur, Nadia-741246, India \\ ${ }^{5}$ Department of Physics, Walter Schottky Institute, and Institute for Advanced Study, \\ Technical University of Munich, 85748 Garching, Germany
}

(Received 20 September 2016; revised manuscript received 27 March 2017; published 26 July 2017)

\begin{abstract}
We consider the dynamics of strongly localized systems subject to dephasing noise with arbitrary correlation time. Although noise inevitably induces delocalization, transport in the noise-induced delocalized phase is subdiffusive in a parametrically large intermediate-time window. We argue for this intermediate-time subdiffusive regime both analytically and using numerical simulations on single-particle localized systems. Furthermore, we show that normal diffusion is restored in the long-time limit, through processes analogous to variable-range hopping. With numerical simulations based on Lanczos exact diagonalization, we demonstrate that our qualitative conclusions are also valid for interacting systems in the many-body localized phase.
\end{abstract}

DOI: 10.1103/PhysRevLett.119.046601

The effects of disorder on quantum transport and dynamics have been a topic of longstanding interest $[1,2]$. Both noninteracting [3] and interacting [4-14] systems of electrons in a random potential can get localized by disorder, causing their dc conductivity to vanish in the limit of a fully isolated system. Isolated localized systems not only have vanishing transport coefficients, but also fail to reach thermal equilibrium starting from generic initial conditions [7]. Yet, in any practical situation the system of interest is coupled to a thermalizing environment, which restores equilibrium and transport. The nature of equilibration in the presence of a bath has been a topic of recent interest [15-26]; however, the implications for transport have not yet been investigated in general (but see Refs. [15,21]).

One reason to expect unusual transport properties in imperfectly isolated localized states is that even perfectly isolated localized states exhibit a broad distribution of time scales. This feature was recently noticed as a property of dynamics near the many-body localization (MBL) transition, where transport has been found to be anomalous [27-35]. However, properties such as overlap integrals between localized orbitals also exhibit broad distributions deep in the localized phase [36-38]. Consequently, the interorbital hopping rates induced by the bath are broadly distributed [22-24]. One might expect such broad distributions to have anomalous transport signatures, particularly in one-dimensional systems, where single weak links can blockade transport.

In the present work, we explore this question, for localized systems coupled to generic non-Markovian dephasing noise. The Markovian limit was previously considered in Refs. [22-24]; these works noted a broad distribution of relaxation times, leading to stretched-exponential decay of the "contrast" (as measured in Ref. [9]). We find that slowly fluctuating noise can have even more dramatic effects: for strong disorder and slowly fluctuating noise, we find a large intermediate time window in which the system exhibits anomalous diffusion (Fig. 1). This anomalous regime vanishes in the limit of Markovian noise, and also crosses over to diffusion in the long-time limit. The existence of a subdiffusive regime is notable because we do not explicitly introduce any broad distributions, as opposed to the cases in Ref. [39]. Rather, a broad distribution of hopping rates emerges from the interplay between disorder, quantum localization effects, and noise, as we discuss below. Furthermore, unlike the subdiffusive regime prefiguring the MBL transition [28], the phenomenon we discuss here is present in the noninteracting limit.

Our focus is on free-fermion systems coupled to classical colored noise, as relatively large systems are accessible in numerical simulations for this case. As we discuss and substantiate with numerical simulations, however, our qualitative conclusions can also be adapted to interacting systems in the MBL phase. Moreover, our model can be extended from classical noise to quantum dephasing, using a mapping between these two processes [40].

Model.-We consider noninteracting fermions in one dimension, subject to a static disorder potential and time dependent noise, as described by

$$
H=-J \sum_{\langle i j\rangle} c_{i}^{\dagger} c_{j}+\sum_{i}\left[\epsilon_{i}+\xi_{i}(t)\right] c_{i}^{\dagger} c_{i},
$$

where $J$ represents the tunneling matrix element and $c_{i}^{\dagger}\left(c_{i}\right)$ creates (destroys) an electron on lattice site $i$. The on-site energies $\epsilon_{i}$ are uncorrelated, and are drawn from a Gaussian distribution of width $W$ and zero mean. The noise $\xi_{i}(t)$ is characterized by its strength $\Lambda$ and correlation time $\tau$. We 


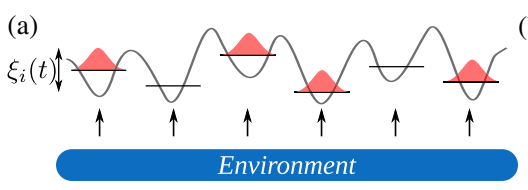

(b)
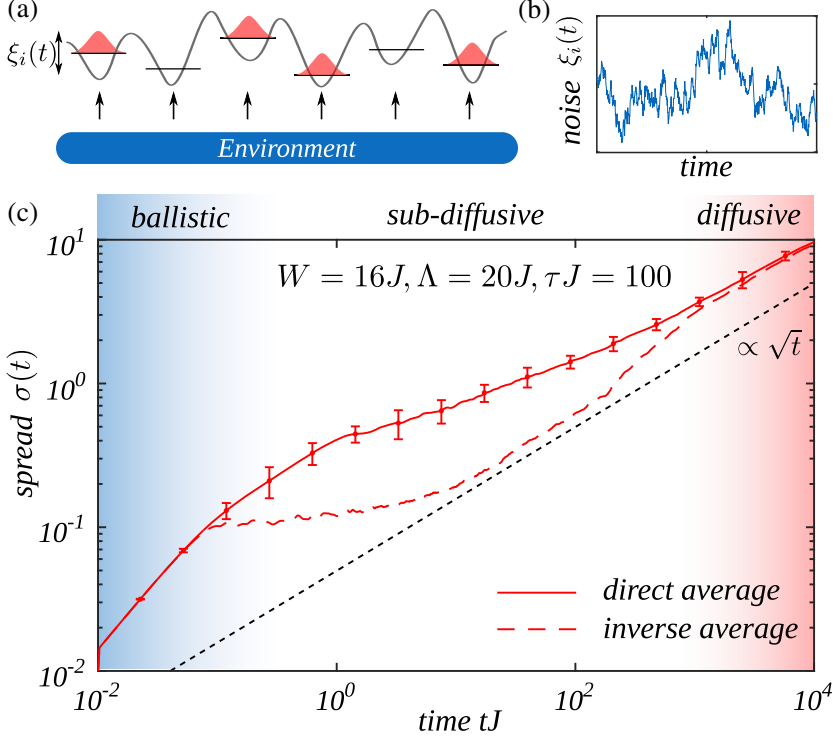

FIG. 1. Noise-induced delocalization. (a) We consider strongly localized fermions in a random potential that are weakly coupled to an environment. (b) In that limit, the environment can be modeled by classical noise $\xi_{i}(t)$ that couples locally to the density. (c) We study the noise-induced transport, by preparing the system in a wave packet and computing its spread $\sigma(t)$ in time. For finite coupling to the environment three regimes can be distinguished: (i) a short-time ballistic expansion $\sigma(t) \sim t$, (ii) a parametrically large regime of subdiffusive transport $\sigma(t) \sim t^{\beta}$ with a continuously increasing power $\beta$ that approaches (iii) a diffusive regime $\sigma(t) \sim \sqrt{t}$ at late times. Numerical data taken as direct (solid) and inverse (dashed) average of the spread over individual realizations are shown for disorder $W=16 \mathrm{~J}$, noise strength $\Lambda=20 J$, and noise correlation time $\tau J=100$.

consider spatially uncorrelated noise generated by an Ornstein-Uhlenbeck process [41] with temporal correlations

$$
C(t)=\left\langle\xi_{i}(t) \xi_{i}(0)\right\rangle=\Lambda^{2} \exp [-|t| / \tau] .
$$

We are interested in how transport changes as a function of the disorder strength $W$ and the noise strength $\Lambda$ as its correlation time is tuned from the Markovian, white noise limit, $\tau \rightarrow 0$, to the limit of quasistatic noise, $\tau \rightarrow \infty$.

Perturbative treatment.-We can analytically explore the noise-induced dynamics, working in the deeply localized limit where the single-particle hopping is the smallest energy scale. (However, the ratios of the other three scales $W, \Lambda, 1 / \tau$ can be arbitrary, so long as each is much larger than $J$.) In this limit, nearest-neighbor hops dominate transport; moreover, the system dephases completely between successive hops, so transport is purely incoherent. Thus, the system can be modeled as a classical one-dimensional hopping model, with rates given by the incoherent nearest-neighbor hopping rate. This rate can be computed through a treatment of a two-site problem [42]. One can solve the equations of motion generated by the Hamiltonian (1) perturbatively in the hopping $J \ll \Lambda, W$. For zero hopping, each site simply accumulates phase, and its wave function amplitude at time $t$, denoted $A_{j}^{0}$, is given by $A_{j}^{0}(t)=A_{j}^{0} e^{-i \epsilon_{i} t-i \phi_{j}(t)}$, where $\phi_{j}(t)=\int_{0}^{t} \xi_{j}\left(t^{\prime}\right) d t^{\prime}$. To describe transport, we expand the equations of motion to the lowest nontrivial order in the hopping, resulting in the following rate equation for the probability distribution $p_{j} \equiv\left|A_{j}\right|^{2}$ for the particle position at time $t$, see Supplemental Material [43],

$\frac{d p_{j}}{d t}=\Gamma_{j, j+1} p_{j+1}+\Gamma_{j, j-1} p_{j-1}-\left(\Gamma_{j+1, j}+\Gamma_{j-1, j}\right) p_{j}$

with a locally varying rate $\Gamma_{i, j}=\Gamma\left(\epsilon_{i}-\epsilon_{j}\right)$ that depends on the energy difference between neighboring sites $i$ and $j$ : $\Gamma(\omega)=2 J^{2} \int_{0}^{\infty} d t \cos (\omega t)\left|C^{\phi}(t)\right|^{2}$, where $C^{\phi}(t)$ is the phase correlation function $C^{\phi}(t)=\left\langle e^{-i \phi_{j}(t)} e^{i \phi_{j}(0)}\right\rangle=$ $e^{-\int_{0}^{t}\left(t-t^{\prime}\right) C\left(t^{\prime}\right) d t^{\prime}}$ with the noise correlation function $C(t)$, Eq. (2), and we have performed a Gaussian average over noise trajectories. For our specific noise model and $\Lambda \tau \gtrsim 1$, the rate $\Gamma(\omega)$ has the form

$\frac{\Gamma(\omega)}{2 J^{2}}= \begin{cases}\frac{\Lambda}{\omega^{2}+\Lambda^{2}} & \omega<\tau^{-1} \\ \Lambda^{-1} e^{-\omega^{2} /\left(4 \Lambda^{2}\right)} & \tau^{-1}<\omega<2 \Lambda \sqrt{\log (\Lambda \tau)} . \\ \frac{\Lambda^{2}}{2 \tau \omega^{4}} & \omega>2 \Lambda \sqrt{\log (\Lambda \tau)}\end{cases}$

Note that Eq. (3) has the form of a random walk with locally varying transition rates. In the disorder-free limit [42], $\Gamma$ has no spatial dependence, and Eq. (3) reduces to a discretized diffusion equation with a diffusion constant $\Gamma(0)$.

Subdiffusive regime.-In the disordered system, the transition rate $\Gamma_{i j}$ between a particular pair of neighboring sites depends on their energy difference $\omega$ through Eq. (4). For very small or very large $\omega$, the rate decreases polynomially with $\omega$. However, in the intermediate regime, which exists only for sufficiently large $\tau, \Gamma(\omega)$ decreases very rapidly as $\omega$ increases. This rapid decrease, as we now discuss, is the origin of anomalous diffusion.

To this end, we estimate the density of very weak links in this regime. Recall that the on-site energies are Gaussian distributed. Then the cumulative distribution function of finding a bottleneck, defined by the transition rate being smaller than a certain threshold $\Gamma_{0}$, follows a power-law relation [43]

$$
P\left(\Gamma<\Gamma_{0}\right) \sim\left(\frac{\Lambda \Gamma_{0}}{2 J^{2}}\right)^{\left(\Lambda^{2} / W^{2}\right)} .
$$

As noted above, we can directly map our problem to a classical rate equation (or resistor network) with random rates (conductances), across each nearest-neighbor link, drawn from the distribution (5). For resistors that are power-law distributed $P(R)=\left(R_{0} / R\right)^{\mu+1}$, the mean resistance is finite for $\mu>1$ (leading to regular diffusion) but ill defined for $\mu<1$ (leading to subdiffusion [28,39]). Our rate distribution corresponds to a heavy-tailed $(\mu<1)$ resistance distribution and thus to subdiffusion when $\Lambda<W$. 
Crossover to diffusion.-Within our noise model, there are two mechanisms that result in a crossover to diffusion at late times. We call these respectively the "variable-range hopping" (VRH) and "ultraviolet" (UV) mechanisms. We begin by discussing the VRH mechanism, which is more generally applicable. This mechanism involves processes that avoid a bottleneck by tunneling virtually through it. Crucially, for a site to act as a bottleneck, all transitions out of it, not just nearest-neighbor hops, must be blocked. The matrix element for an $n$-site virtual process is $J(J / W)^{n-1}$, and the corresponding incoherent rate is given by $\Gamma_{i}^{(n)} \simeq$ $\left(2 J^{2} / \Lambda\right)(J / W)^{2(n-1)} \exp \left[-\left(\omega^{2} / 4 \Lambda^{2}\right)\right]$. For a site to act as a bottleneck we require that $\prod_{n} \Gamma_{i}^{(n)} \lesssim \Gamma_{0}$; i.e., each link must independently act as a bottleneck. In effect, this product only runs over $n \leq n^{*}=\log \left(\Gamma_{0} \Lambda / 2 W^{2}\right) / 2 \log (J / W)$, as more distant links are slower than $\Gamma_{0}$ regardless of the energy difference $\omega$ [43]. The probability of finding a series of such sites can be estimated (Supplemental Material [43]) as

$$
\tilde{P}\left(\Gamma_{0} \mid n^{*}\right) \sim \exp \left(-c \log ^{2} \frac{\Gamma_{0} \Lambda}{2 W^{2}}\right),
$$

with a constant $c \simeq \Lambda^{2} /\left(4 W^{2} \log [W / J]\right)$. This probability decays slightly faster than a power law in $1 / \Gamma_{0}$ and, hence, bottlenecks are asymptotically always sufficiently rare such that diffusion is recovered. Specifically, as the mean inverse transition rate (i.e., resistance) is well defined, we can compute the asymptotic diffusion constant by taking the inverse of this mean resistance. For $W \gg \Lambda$ we find [43]

$$
D_{\mathrm{VRH}} \simeq \frac{W}{\sqrt{\pi \log [W / J]}}\left(\frac{J}{W}\right)^{W^{2} / \Lambda^{2}} .
$$

We now turn to the ultraviolet mechanism. Within our noise model (2), the correlation function (4) crosses over from a Gaussian to a power law, $1 / \omega^{4}$, at large frequencies $\omega>2 \Lambda \sqrt{\log (\Lambda \tau)}$. The incoherent transition rate for pairs of sites with detuning $\left|\epsilon_{i}-\epsilon_{j}\right|>2 \Lambda \sqrt{\log (\Lambda \tau)}$ is not suppressed strongly with their detuning, and they do not bottleneck transport. The overall diffusion constant is set by the weakest common links, for which $\omega \simeq 2 \Lambda \sqrt{\log \Lambda \tau}$. The density of these links is $\exp \left[-\Lambda^{2} \log (\Lambda \tau) / 2 W^{2}\right]$, and the rate across each is $2 J^{2} /\left(\Lambda^{2} \tau\right)$. Thus the effective UV diffusion constant scales as

$$
D_{\mathrm{UV}} \simeq \frac{2 J^{2}}{\Lambda} \frac{1}{(\Lambda \tau)^{1-(\Lambda / W)^{2}}} .
$$

Within our noise model, the asymptotic diffusion coefficient is set by $\max \left(D_{\mathrm{VRH}}, D_{\mathrm{UV}}\right)$. However, the power-law regime in (4) originates from the "cuspy" short-time behavior of the noise correlation function (2). This feature is model dependent, and indeed is absent for noise generated, e.g., by the dynamics of a finite-bandwidth quantum system. In systems where the noise correlation function is analytic at short times, diffusion is solely due to the VRH mechanism.

Numerical results.-We quantitatively study the subdiffusive transport by performing exact numerical simulations of a particle localized in the center of our system. We first compute stochastic noise trajectories based on the Ornstein-Uhlenbeck process. Second, we numerically solve the equations of motion set by Hamiltonian (1). We consider systems of size $L=400$ and times to $t J=$ $10^{4}$. A typical example for the spread $\sigma(t)=\sqrt{\left\langle\hat{x}^{2}\right\rangle-\langle\hat{x}\rangle^{2}}$ is shown in Fig. 1(c). Here, the expectation values $\langle\cdots\rangle$ are taken with respect to the time evolved wave function $|\psi(t)\rangle$. At times $t J \lesssim 1$, the expansion of the wave packet is ballistic. At later times the spread crosses over to subdiffusive behavior $\sigma(t) \sim t^{\beta}$. In that regime, the direct sample average of the spread over disorder and noise realizations $\langle\sigma(t)\rangle$, solid line, and the inverse average of the inverse spread $1 /\left\langle\sigma^{-1}(t)\right\rangle$, dashed lines, strongly disagree. This is a manifestation of the probability distribution (5) having ill-defined moments. The apparent subdiffusion exponent $\beta$ increases with time and slowly approaches the diffusive limit $\beta=1 / 2$ at late times $t J \sim 10^{4}$.

Simulations of the wave-packet spread $\sigma(t)$ are shown in Fig. 2 for a range of parameters. Generally, we observe (i) an initial ballistic expansion, followed by (ii) an intermediate subdiffusive regime that gradually crosses over to (iii) diffusion. With increasing disorder, $\sigma(t)$ decreases and the crossover to diffusive transport is pushed
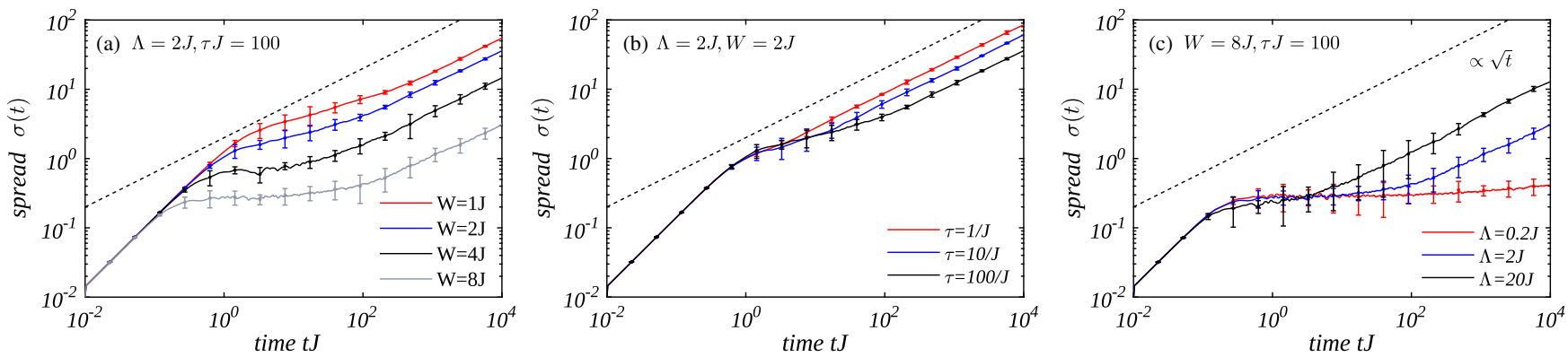

FIG. 2. Time evolution of a localized wave packet. The numerically evaluated, inverse-averaged spread $\sigma(t)$ of an initially localized wave packet for systems of size $L=400$ is shown for (a) fixed $\Lambda=2 J, \tau J=100$, (b) $\Lambda=2 J, W=2 J$, and (c) $W=8 J, \tau J=100$. Dotted lines indicate diffusive expansion $\sigma(t) \sim \sqrt{t}$. Error bars are obtained from the sample average of 250 noise and disorder realizations. 


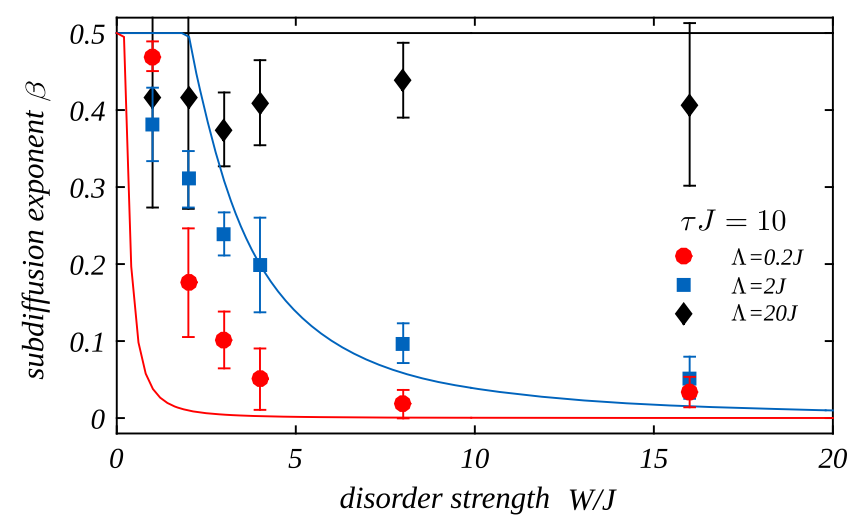

FIG. 3. Short-time subdiffusion exponent. The power-law exponent $\beta$ characterizing the initial subdiffusive transport $\sigma(t) \sim$ $t^{\beta}$ is extracted from fitting the numerical data in the range $1<$ $t J<\tau J$ for $\tau J=10$. In the weak noise limit $\Lambda \lesssim J$, the exponent depends strongly on the disorder strength $W$ approaching 0 with increasing $W$ whereas it is constant for strong noise $\Lambda=20 J$. For weak disorder strength $W \rightarrow 0, \beta$ universally approaches within the error bars the limit of diffusive transport with $\beta=1 / 2$. Solid lines indicate our estimate $\beta=\Lambda^{2} /\left(\Lambda^{2}+W^{2}\right)$.

to later times, Fig. 2(a). Moreover, with increasing noise correlation time $\tau$, the intermediate subdiffusive regime is extended, leading to a decrease of the asymptotic diffusion constant with $\tau$, Fig. 2(b). This suggests that for the relevant parameters subdiffusion is cut off by the ultraviolet mechanism (8). Finally, at strong disorder, transport is facilitated with increasing noise strength, Fig. 2(c). At weak disorder, however, noise impedes transport (data not shown). These qualitative findings are fully consistent with expectations from perturbation theory.

We evaluate the subdiffusion exponent $\sigma(t) \sim t^{\beta}$ by fitting the numerical data in the regime $1<t J<\tau J$, Fig. 3. The small range is chosen to capture the exponent at the onset of the subdiffusive regime. For weak noise and strong disorder, the subdiffusion exponent $\beta$ is near 0 . When lowering the disorder strength, $\beta$ strongly increases and approaches the diffusive limit $\beta \rightarrow 1 / 2$. By contrast at large disorder, $\beta$ sets off at a larger value and quickly saturates. We obtain an estimate for $\beta$ by relating it to the exponent of the cumulative distribution function (5) as $\beta=$ $\Lambda^{2} /\left(\Lambda^{2}+W^{2}\right)$ [28], indicated by solid lines in Fig. 3. The data qualitatively reproduce the predicted trend but slight quantitative differences are present. Such discrepancies are not unexpected as our theoretical analysis is valid to lowest order in $J / W, J / \Lambda$, and these parameters are not small in the numerically accessible regime.

From the long-time asymptotics of the spread $\sigma(t)$, we extract the diffusion constant $\sigma(t \rightarrow \infty)=\sqrt{2 D t}$ for different values of the noise and disorder strength at fixed noise correlation time $\tau J=1$ (Fig. 4). For strong noise compared with disorder, the diffusion constant is largely disorder independent, and decreases with increasing noise as $\sim 1 / \Lambda$, Eq. (4), consistent with Refs. [42,44]. In the

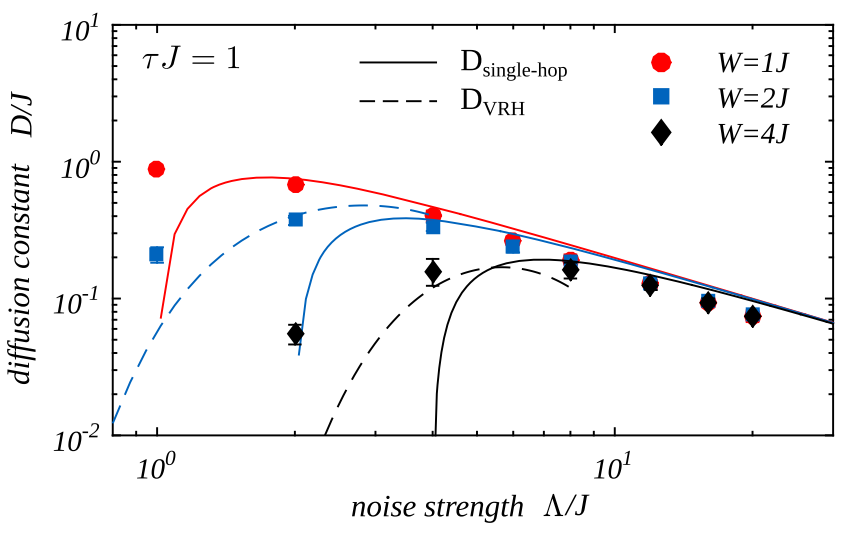

FIG. 4. Asymptotic diffusion constant. The diffusion constant $D$ evaluated from the asymptotic spread of the wave packet $\sigma(t \rightarrow \infty)=\sqrt{2 D t}$ is shown as a function of the noise strength $\Lambda$ for different values of the disorder strength $W$ and fixed noise correlation time $\tau J=1$, symbols. The numerical data are compared to the single-hop model valid for $\Lambda \gtrsim W$, solid lines, and the variable-range hopping model of Eq. (7) valid for $\Lambda \lesssim W$, dashed lines.

strong noise limit, $\Lambda \gg W$, diffusion is induced already by nearest-neighbor hops, leading to $D_{\text {singlex hop }} \sim$ $2 J^{2}\left(1-W^{2} / \Lambda^{2}\right) / \Lambda$ (solid lines) [43]. In the subdiffusive regime, $\Lambda<W$, it is challenging to propagate to sufficiently long times to see the eventual crossover to diffusion. However, we were able to extract a few data points in that limit, and observe a reversed dependence: noise assists diffusion rather than impeding it as predicted by the variable range processes, Eq. (7) (dashed lines).

Discussion.- - How robust are our conclusions to adding interactions, and to more general forms of correlated noise? Adapting our results to interacting, many-body localized systems coupled to noise is straightforward in principle. Qualitatively, the main difference is that there are many more ways for an interacting system to "escape" a bottleneck: in addition to longer-range hops, the system can undergo manyparticle rearrangements, which have a larger phase space [36]. Thus the variable-range hopping mechanism is more effective, giving rise to a smaller subdiffusive window and a larger asymptotic diffusion constant. The Supplemental Material supports these expectations by studying transport in interacting and localized systems using numerical simulations based on Lanczos exact diagonalization [43].

Interacting localized systems coupled to Markovian baths have been shown to exhibit a stretched-exponential decay of the contrast of an initial density-wave pattern [22-24]. We find similar behavior in our system with a stretching exponent that is independent of the noise correlation time $\tau$ for weak noise $\Lambda \lesssim J$ but depends strongly on $\tau$ for large noise $\Lambda \gtrsim J$ [43].

Our perturbative analysis suggests that our numerical results should be sensitive to the short-time correlations of the noise, which are nonuniversal. In particular, noise emanating from a physical system with a finite bandwidth 
decays as a Gaussian, rather than an exponential, on time scales that are short compared with the bandwidth. Thus, the ultraviolet mechanism should be absent in such systems. This interdependence of slow and fast processes has also been observed in a mean-field treatment of the MBL transition [17], and appears to be a generic phenomenon, reminiscent of "UV-IR mixing" in field theory [45]. Extending our numerical studies to more general forms of colored noise is an important direction, which we explore in future work.

Outlook.-We have studied noise-induced transport in disordered quantum systems. We have argued that for slowly fluctuating noise, transport is governed by an incoherent hopping model with an emergent broad distribution of hopping rates, causing anomalous diffusion on intermediate time scales, and regular diffusion (with a strongly suppressed diffusion constant) at late times. The subdiffusive regime is parametrically large when the noise correlation time $\tau$ is long, so that $\Lambda \ll W \ll \Lambda \sqrt{\log (\Lambda \tau)}$. As this regime grows slowly with $\tau$, numerical simulations cannot access the regime where perturbation theory is quantitatively reliable. Nevertheless, simulations clearly show the predicted trends, specifically subdiffusive intermediate-time dynamics and a late-time crossover to diffusion.

Our approach paves the way for developing a selfconsistent theory for the thermal phase in disordered interacting quantum systems where interactions can be treated by a self-consistent Hartree-Fock decoupling. It has been argued that, within a Hartree-Fock treatment, interacting and disordered bosons exhibit subdiffusive transport [46-48]. Hence, it would be interesting to classify the effective noise spectrum in such mean-field bosonic systems and analyze the anomalous transport using the framework presented here. Furthermore, such a self-consistent theory can also be developed with the prospect of studying the response of a fully many-body localized system coupled to a bath. Having technical approaches at hand, which go beyond conventional exact diagonalization of small quantum systems, helps to provide further insight in the many-body localized phase and its breakdown.

We thank K. Agarwal, A. Amir, S. Choi, E. Demler, M. Lukin, V. Oganesyan, and W. Witt for many useful discussions. We acknowledge support from Technical University of Munich-Institute for Advanced Study, funded by the German Excellence Initiative and the European Union FP7 under Grant No. 291763, from the DFG Grant No. KN 1254/1-1, and the Walter Burke Foundation at Caltech.

[1] P. A. Lee and T. V. Ramakrishnan, Disordered electronic systems, Rev. Mod. Phys. 57, 287 (1985).

[2] B. Kramer and A. MacKinnon, Localization: Theory and experiment, Rep. Prog. Phys. 56, 1469 (1993).
[3] P. W. Anderson, Absence of diffusion in certain random lattices, Phys. Rev. 109, 1492 (1958).

[4] L. Fleishman and P. W. Anderson, Interactions and the anderson transition, Phys. Rev. B 21, 2366 (1980).

[5] D. M. Basko, I. L. Aleiner, and B. L. Altshuler, Metalinsulator transition in a weakly interacting many-electron system with localized single-particle states, Ann. Phys. (Amsterdam) 321, 1126 (2006).

[6] I. V. Gornyi, A. D. Mirlin, and D. G. Polyakov, Interacting Electrons in Disordered Wires: Anderson Localization and Low-t Transport, Phys. Rev. Lett. 95, 206603 (2005).

[7] R. Nandkishore and D. A. Huse, Many-body localization and thermalization in quantum statistical mechanics, Annu. Rev. Condens. Matter Phys. 6, 15 (2015).

[8] E. Altman and R. Vosk, Universal dynamics and renormalization in many body localized systems, Annu. Rev. Condens. Matter Phys. 6, 383 (2015).

[9] M. Schreiber, S. S. Hodgman, P. Bordia, H. P. Lüschen, M. H. Fischer, R. Vosk, E. Altman, U. Schneider, and I. Bloch, Observation of many-body localization of interacting fermions in a quasirandom optical lattice, Science 349, 842 (2015)

[10] S. S. Kondov, W. R. McGehee, W. Xu, and B. DeMarco, Disorder-Induced Localization in a Strongly Correlated Atomic Hubbard Gas, Phys. Rev. Lett. 114, 083002 (2015).

[11] J. Smith, A. Lee, P. Richerme, B. Neyenhuis, P. W. Hess, P. Hauke, M. Heyl, D. A. Huse, and C. Monroe, Many-body localization in a quantum simulator with programmable random disorder, Nat. Phys. 12, 907 (2016).

[12] P. Bordia, H. P. Lüschen, S. S. Hodgman, M. Schreiber, I. Bloch, and U. Schneider, Coupling Identical OneDimensional Many-Body Localized Systems, Phys. Rev. Lett. 116, 140401 (2016).

[13] J.-y. Choi, S. Hild, J. Zeiher, P. Schauß, A. Rubio-Abadal, T. Yefsah, V. Khemani, D. A. Huse, I. Bloch, and C. Gross, Exploring the many-body localization transition in two dimensions, Science 352, 1547 (2016).

[14] P. Bordia, H. Lüschen, U. Schneider, M. Knap, and I. Bloch, Periodically driving a many-body localized quantum system, Nat. Phys. 13, 460 (2017).

[15] D. M. Basko, I. L. Aleiner, and B. L. Altshuler, Possible experimental manifestations of the many-body localization, Phys. Rev. B 76, 052203 (2007).

[16] R. Nandkishore, S. Gopalakrishnan, and D. A. Huse, Spectral features of a many-body-localized system weakly coupled to a bath, Phys. Rev. B 90, 064203 (2014).

[17] S. Gopalakrishnan and R. Nandkishore, Mean-field theory of nearly many-body localized metals, Phys. Rev. B 90, 224203 (2014).

[18] S. Johri, R. Nandkishore, and R. N. Bhatt, Many-Body Localization in Imperfectly Isolated Quantum Systems, Phys. Rev. Lett. 114, 117401 (2015).

[19] D. A Huse, R. Nandkishore, F. Pietracaprina, V. Ros, and A. Scardicchio, Localized systems coupled to small baths: From anderson to zeno, Phys. Rev. B 92, 014203 (2015).

[20] K. Hyatt, J. R. Garrison, A. C. Potter, and B. Bauer, Manybody localization in the presence of a small bath, Phys. Rev. B 95, 035132 (2017). 
[21] S. A. Parameswaran and S. Gopalakrishnan, Spin-catalyzed hopping conductivity in disordered strongly interacting quantum wires, Phys. Rev. B 95, 024201 (2017).

[22] M. H. Fischer, M. Maksymenko, and E. Altman, Dynamics of a Many-Body-Localized System Coupled to a Bath, Phys. Rev. Lett. 116, 160401 (2016).

[23] E. Levi, M. Heyl, I. Lesanovsky, and J. P. Garrahan, Robustness of Many-Body Localization in the Presence of Dissipation, Phys. Rev. Lett. 116, 237203 (2016).

[24] M. V. Medvedyeva, T. Prosen, and M. Žnidarić, Influence of dephasing on many-body localization, Phys. Rev. B 93, 094205 (2016).

[25] B. Everest, I. Lesanovsky, J. P. Garrahan, and E. Levi, Role of interactions in a dissipative many-body localized system, Phys. Rev. B 95, 024310 (2017).

[26] R. Nandkishore and S. Gopalakrishnan, General theory of many-body-localized systems coupled to baths, Ann. Phys. (Amsterdam) (to be published).

[27] Y. BarLev, G. Cohen, and D. R. Reichman, Absence of Diffusion in an Interacting System of Spinless Fermions on a One-Dimensional Disordered Lattice, Phys. Rev. Lett. 114, 100601 (2015).

[28] K. Agarwal, S. Gopalakrishnan, M. Knap, M. Müller, and E. Demler, Anomalous Diffusion and Griffiths Effects Near the Many-Body Localization Transition, Phys. Rev. Lett. 114, 160401 (2015).

[29] R. Vosk, D. A. Huse, and E. Altman, Theory of the ManyBody Localization Transition in One-Dimensional Systems, Phys. Rev. X 5, 031032 (2015).

[30] A. C. Potter, R. Vasseur, and S. A. Parameswaran, Universal Properties of Many-Body Delocalization Transitions, Phys. Rev. X 5, 031033 (2015).

[31] D. J. Luitz, N. Laflorencie, and F. Alet, Extended slow dynamical regime close to the many-body localization transition, Phys. Rev. B 93, 060201 (2016).

[32] S. Gopalakrishnan, K. Agarwal, E. A. Demler, D. A. Huse, and M. Knap, Griffiths effects and slow dynamics in nearly many-body localized systems, Phys. Rev. B 93, 134206 (2016).

[33] K. Agarwal, E. Altman, E. Demler, S. Gopalakrishnan, D. A. Huse, and M. Knap, Rare region effects and dynamics near the many-body localization transition, Ann. Phys. (Amsterdam) 529, 1600326 (2017).

[34] H. P. Lüschen, P. Bordia, S. Scherg, F. Alet, E. Altman, U. Schneider, and I. Bloch, Evidence for Griffiths-type dynamics near the many-body localization transition in quasi-periodic systems, arXiv:1612.07173.
[35] P. Bordia, H. P. Lüschen, S. Scherg, S. Gopalakrishnan, M. Knap, U. Schneider, and I. Bloch, Probing slow relaxation and many-body localization in two-dimensional quasi-periodic systems, arXiv:1704.03063.

[36] S. Gopalakrishnan, M. Müller, V. Khemani, M. Knap, E. Demler, and D. A. Huse, Low-frequency conductivity in many-body localized systems, Phys. Rev. B 92, 104202 (2015).

[37] D. Pekker, B. K. Clark, V. Oganesyan, and G. Refael, Fixed points of Wegner-Wilson Flows and Many-Body Localization, arXiv:1607.07884.

[38] M. Serbyn, M. Knap, S. Gopalakrishnan, Z. Papić, N. Y. Yao, C. R. Laumann, D. A. Abanin, M. D. Lukin, and E. A. Demler, Interferometric Probes of Many-Body Localization, Phys. Rev. Lett. 113, 147204 (2014).

[39] J.-P. Bouchaud and A. Georges, Anomalous diffusion in disordered media: Statistical mechanisms, models and physical applications, Phys. Rep. 195, 127 (1990).

[40] D. Crow and R. Joynt, Classical simulation of quantum dephasing and depolarizing noise, Phys. Rev. A 89, 042123 (2014).

[41] C. W. Gardiner et al., Handbook of Stochastic Methods (Springer, Berlin, 1985), Vol. 3.

[42] A. Amir, Y. Lahini, and H. B. Perets, Classical diffusion of a quantum particle in a noisy environment, Phys. Rev. E 79, 050105 (2009).

[43] See Supplemental Material http://link.aps.org/supplemental/ 10.1103/PhysRevLett.119.046601 for details on the perturbative treatment, the crossover from subdiffusion to diffusion, the evolution of the imbalance, and noise-induced dynamics in many-body localized systems.

[44] M. Žnidarič and M. Horvat, Transport in a disordered tight-binding chain with dephasing, Eur. Phys. J. B 86, 67 (2013).

[45] S. Minwalla, M. V. Raamsdonk, and N. Seiberg, Noncommutative perturbative dynamics, J. High Energy Phys. 02 (2000) 020.

[46] E. Lucioni, B. Deissler, L. Tanzi, G. Roati, M. Zaccanti, M. Modugno, M. Larcher, F. Dalfovo, M. Inguscio, and G. Modugno, Observation of Subdiffusion in a Disordered Interacting System, Phys. Rev. Lett. 106, 230403 (2011).

[47] D. L. Shepelyansky, Delocalization of Quantum Chaos by Weak Nonlinearity, Phys. Rev. Lett. 70, 1787 (1993).

[48] S. Flach, D. O. Krimer, and Ch. Skokos, Universal Spreading of Wave Packets in Disordered Nonlinear Systems, Phys. Rev. Lett. 102, 024101 (2009). 